\title{
Structural, optical and morphological studies of undoped and Zn-doped CdSe QDs via aqueous route synthesis
}

\author{
N THIRUGNANAM and D GOVINDARAJAN* \\ Department of Physics, Annamalai University, Annamalai Nagar 608 002, India
}

MS received 10 June 2015; accepted 10 May 2016

\begin{abstract}
Undoped and Zn-doped CdSe quantum dots (QDs) were successfully synthesized by the chemical precipitation method. The structural, optical and morphological properties of the synthesized undoped and $\mathrm{Zn}$-doped CdSe QDs were studied by X-ray diffraction (XRD), UV-visible absorption spectroscopy, photoluminescence (PL) spectroscopy, fluorescence lifetime spectroscopy, scanning electron microscopy (SEM), field emission transmission electron microscopy (FE-TEM) and FTIR. The synthesized undoped and Zn-doped CdSe QDs were in cubic crystalline phase, which was confirmed by the XRD technique. From the UV-visible absorption spectral analysis, the absorption wavelengths of both undoped and Zn-doped CdSe QDs show blue-shift with respect to their bulk counterpart as a result of quantum confinement effect. The highest luminescence intensity was observed for CdSe QDs doped with $4 \% \mathrm{Zn}$ by PL studies. TEM analysis shows that the prepared QDs are spherical in shape.
\end{abstract}

Keywords. CdSe QDs; cubic crystalline phase; quantum confinement effect; luminescence.

\section{Introduction}

Presently, semiconductor quantum dots (QDs) are attractive to the scientific community due to their unique optical and electronic properties, which cannot be obtained from their bulk counterparts $[1,2]$. Among the different semiconductor materials, CdSe is one of the interesting materials belonging to the II-VI group with direct bandgap energy of $1.74 \mathrm{eV}$ at $300 \mathrm{~K}$ [3]. Due to quantum confinement effect, $\mathrm{CdSe}$ QDs show a strong fluorescence and can continuously be tuned according to the particle size. Scientists have taken many efforts to control the electronic structures of QDs by changing their size, shape and composition. CdSe QDs find potential applications widely in different areas such as solar cells [4,5], light-emitting diodes [6], photocatalysis [7], biological labels and bioimaging [8-10], biosensing [11] and chemosensing [12]. Also, doping the CdSe semiconductor QDs with $\mathrm{Zn}$ ions has been the new era of research and this combination forms a new class of luminescent materials. The divalent $\mathrm{Zn}^{2+}$ transition metal ions replace $\mathrm{Cd}^{2+}$ ions in their lattice sites of CdSe, which leads to the energy level modification. Synthesis of $\mathrm{Zn}$-doped CdSe nanoparticles using hydrothermal method and its photocatalytic properties were studied by Song and Zhang [13]. Generally, the synthesis method for CdSe QDs can be broadly classified into two categories, namely organic route [14-16] and aqueous route [17-19]. When compared with aqueous route, organic route synthesis method yields high crystallinity QDs but this method operates at high temperature using dimethyl cadmium, trioctylphosphine (TOP) and trioctylphosphine oxide

\footnotetext{
*Author for correspondence (degerajan@gmail.com)
}

(TOPO) as precursors; they are extremely toxic, expensive and pyrophoric in nature. Hence researchers mostly use the inexpensive, less toxic aqueous method for preparation of CdSe QDs. But the luminescence properties of Zn-doped CdSe QDs synthesized by chemical precipitation method were reported rarely.

In the present work, a simple and inexpensive aqueous method was used for synthesizing undoped and $\mathrm{Zn}$-doped $\mathrm{CdSe}$ QDs at moderate temperature without any capping agents.

\section{Materials and methods}

\subsection{Materials}

Cadmium acetate dihydrate $\left(\mathrm{Cd}\left(\mathrm{CH}_{3} \mathrm{COO}\right)_{2} \cdot 2 \mathrm{H}_{2} \mathrm{O}\right)$, selenium (Se), sodium sulphite $\left(\mathrm{Na}_{2} \mathrm{SO}_{3}\right)$ and zinc acetate dihydrate $\left(\mathrm{Zn}\left(\mathrm{CH}_{3} \mathrm{COO}\right)_{2} \cdot 2 \mathrm{H}_{2} \mathrm{O}\right)$ were purchased from $\mathrm{S}$ D Fine-Chem Limited, India. All reagents were of analytical grade and used without further purification. The entire work was carried out using deionized (DI) water.

\subsection{Synthesis of undoped and Zn-doped CdSe QDs}

Sodium sulphite $(0.03 \mathrm{~mol})$ and 5 -mmol selenium were added in 100-ml DI water in a 250-ml three-neck flask. The solution was refluxed and deaerated by nitrogen gas for $6 \mathrm{~h}$ at $80^{\circ} \mathrm{C}$; finally sodium selenosulphate solution formed.

Cadmium acetate dihydrate $(4 \mathrm{mmol})$ was dissolved in 50 -ml DI water. To this solution, $100 \mathrm{ml}$ of sodium selenosulphite solution was added drop by drop. The entire solution was stirred and heated at $80^{\circ} \mathrm{C}$ for $3 \mathrm{~h}$. At the end of the reaction, red-coloured $\mathrm{CdSe}$ solution formed and it 
was precipitated. The precipitate was centrifuged and then washed twice with water and ethanol for removing impurities present in it. For $\mathrm{Zn}$-doped $\mathrm{CdSe}$, zinc acetate dihydrate of different concentrations (1-5\%) was added to the cadmium acetate dihydrate solution and then the same procedure was followed.

\subsection{Characterization techniques}

The X-ray diffraction (XRD) pattern of powdered sample was recorded using an X'PERT-PRO diffractometre with $\mathrm{CuK}_{\alpha}(1.54060 \AA)$ at room temperature. The optical absorption and photoluminescence spectra of CdSe QDs were recorded using a SHIMADZU-UV 1800 spectrophotometer and a Perkin-Elmer LS55 fluorescence spectrometer, respectively. The fluorescence lifetime measurements were recorded using a nanosecond-time-correlated single-photon-counting spectrometer Horiba Fluorocube-01-NL lifetime system with a nano-LED (pulsed diode excitation source) as the excitation source with the excitation wavelength of $282 \mathrm{~nm}$ and a TBXPS as the detector. The morphology of the QDs was studied using scanning electron microscopy (SEM: JEOL, JSM-5610 $\mathrm{LV})$. The field emission transmission electron microscopy (FE-TEM) study was carried out using a JSM-2100F JEOI field emission microscope. The FT-IR spectra were recorded from a SHIMADZU-8400 with a resolution of $4 \mathrm{~cm}^{-1}$.

\section{Results and discussion}

\subsection{XRD study}

The crystalline structures of the prepared undoped and Zndoped CdSe QDs were studied by X-ray diffraction technique and are shown in figure 1 . All the diffraction peaks correspond to (111), (220) and (311) planes of cubic zinc blende structure (JCPDS: 19-0191). This crystalline phase is often observed for CdSe prepared in aqueous solution [2022]. The broad peaks observed in XRD patterns clearly indicate that the prepared samples are nanodimensional. From the XRD study, there is no change in crystalline phase for $\mathrm{Zn}$-doped samples; this is due to the incorporation of $\mathrm{Zn}^{2+}$ ions in $\mathrm{Cd}^{2+}$ lattice sites. No peaks corresponding to impurities were detected, indicating the high purity of the product. The average crystallite size is calculated from the Debye-Scherrer formula

$$
D=\frac{0.9 \lambda}{\beta \cos \theta},
$$

where $k$ is a constant (about 0.9$), \lambda=1.54060 \mathrm{~s}\left(\mathrm{Cu} \mathrm{K}_{\alpha}\right.$ radiation wavelength), $\beta$ is full-width at half-maximum and $\theta$ the Bragg diffraction angle. The calculated crystallite sizes and lattice constants for both samples are presented in table 1. The calculated crystallite size for undoped CdSe QDs is $4.4 \mathrm{~nm}$ and $\mathrm{Zn}$-doped CdSe QDs are in the range 4.1-3.5 $\mathrm{nm}$. The Zn-doped CdSe QDs crystallite size decreases as $\mathrm{Zn}$ doping concentration increases and it is

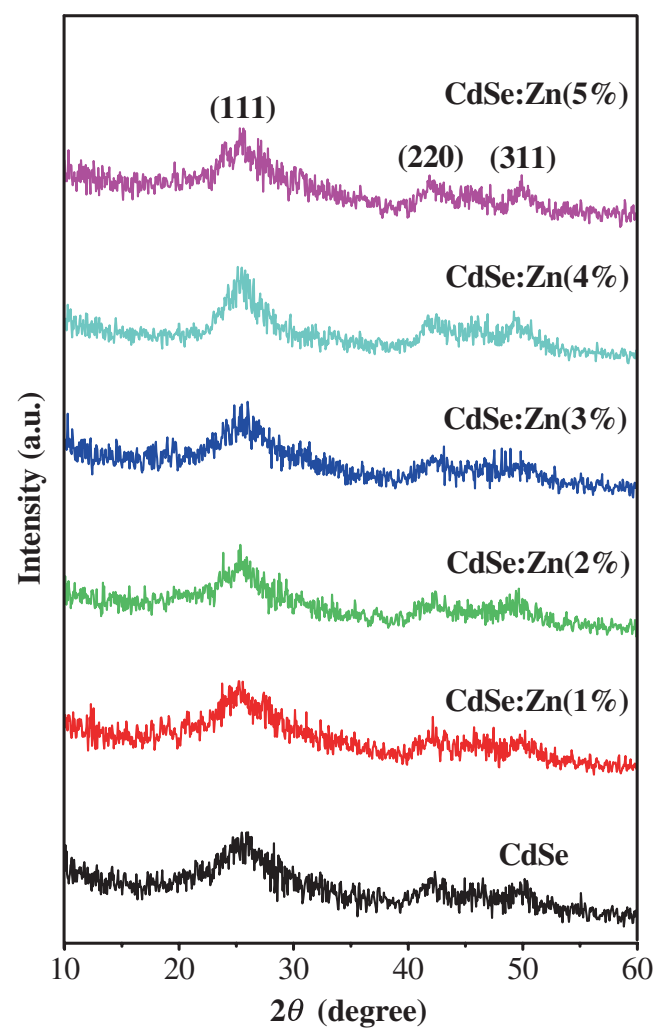

Figure 1. XRD spectra for undoped and Zn-doped CdSe QDs.

Table 1. Crystallite sizes and lattice constants of undoped and Zn-doped CdSe QDs.

\begin{tabular}{lcc}
\hline $\begin{array}{l}\text { Doping } \\
\text { concentration (\%) }\end{array}$ & $\begin{array}{c}\text { Crystallite } \\
\text { sizes (nm) }\end{array}$ & $\begin{array}{c}\text { Lattice } \\
\text { constants }\left(10^{-10} \mathrm{~m}\right)\end{array}$ \\
\hline CdSe & 4.4 & 6.0630 \\
CdSe:Zn(1\%) & 4.1 & 6.0626 \\
CdSe:Zn(2\%) & 3.8 & 6.0612 \\
CdSe:Zn(3\%) & 3.7 & 6.0595 \\
CdSe:Zn(4\%) & 3.6 & 6.0584 \\
CdSe:Zn $(5 \%)$ & 3.5 & 6.0571 \\
\hline
\end{tabular}

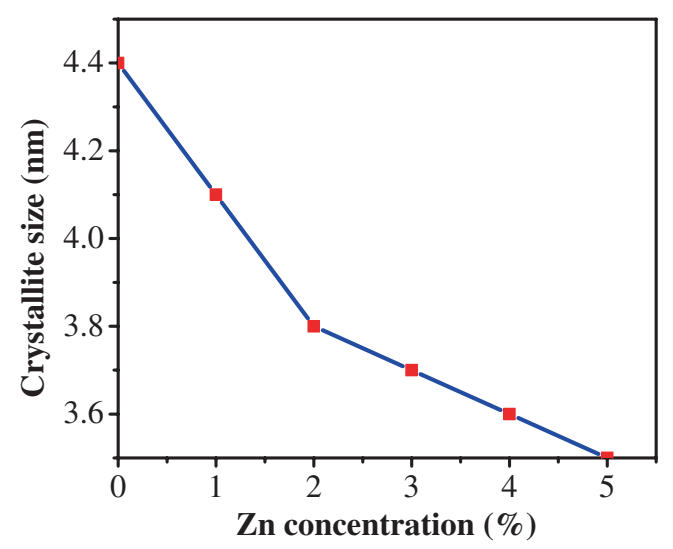

Figure 2. $\mathrm{Zn}$ concentration (\%) vs. crystallite size (nm) for undoped and Zn-doped CdSe QDs. 


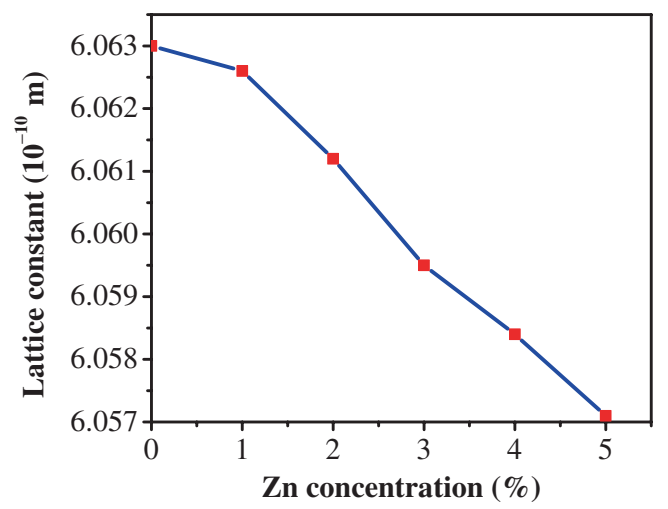

Figure 3. Zn concentration (\%) vs. lattice constant of undoped and Zn-doped CdSe QDs.

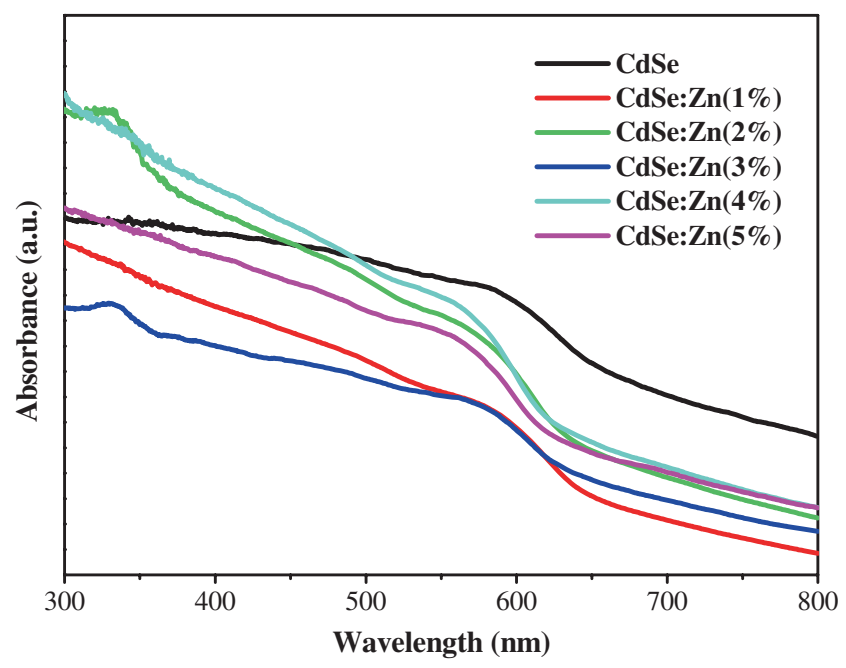

Figure 4. UV-visible absorption spectra for undoped and Zndoped CdSe QDs.

shown in figure 2. The calculated lattice constant for undoped CdSe QDs is $6.063 \AA$ and for CdSe: $\mathrm{Zn}(4 \%)$ it is $6.0584 \AA$ (figure 3 ). The decrease in lattice constant is due to replacement of $\mathrm{Cd}^{2+}$ ions by $\mathrm{Zn}^{2+}$ ions, since the ionic radius of $\mathrm{Cd}^{2+}(0.92 \mathrm{~nm})$ is greater than that of $\mathrm{Zn}^{2+}(0.74 \mathrm{~nm})$.

\subsection{UV-visible absorption studies}

Figure 4 shows the UV-visible absorption spectra of undoped and $\mathrm{Zn}$ doped CdSe QDs. The absorption peaks for undoped CdSe QDs is located at $583 \mathrm{~nm}$, and at 574, 566, 562, 558 and $555 \mathrm{~nm}$ for Zn-doped CdSe QDs. For bulk CdSe, direct bandgap is $1.74 \mathrm{eV}$ and an absorption peak therefore occurred at $716 \mathrm{~nm}$ [23]. Thus there is a strong blue-shift in the spectra, indicating that particles must be smaller than the Bohr radius of excitons, which is $5.6 \mathrm{~nm}$ for CdSe [24].

The energy gaps for undoped and Zn-doped CdSe QDs were calculated according to the relation [25]

$$
E=h v \text {. }
$$

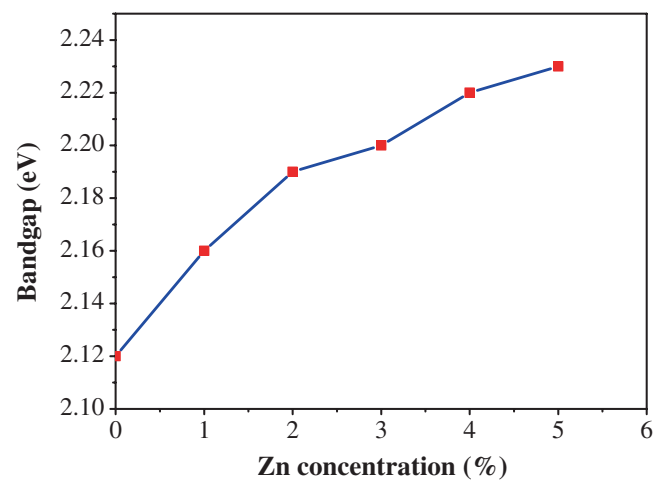

Figure 5. Zn concentration (\%) vs. bandgap (eV) for undoped and Zn-doped CdSe QDs.

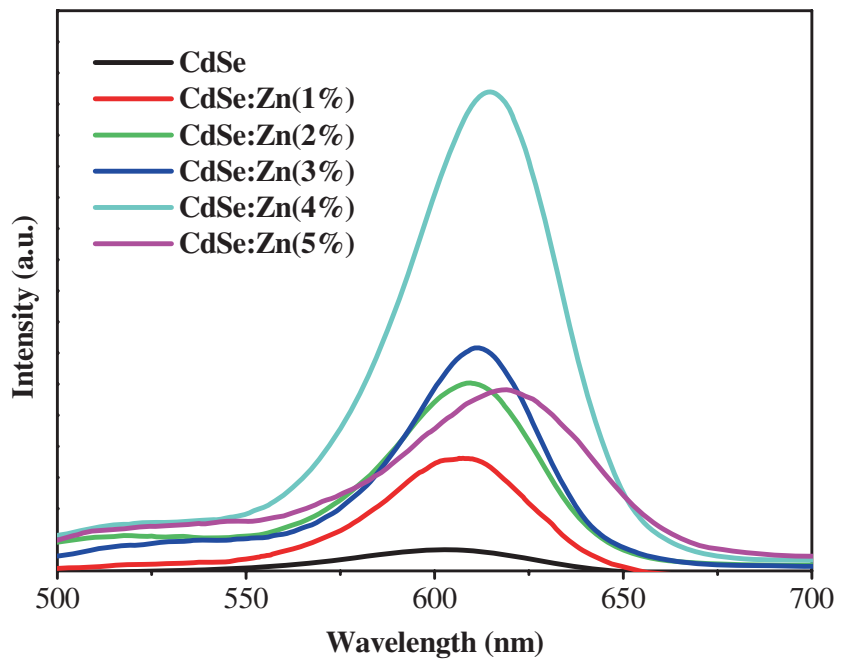

Figure 6. PL spectra for undoped and Zn-doped CdSe QDs.

The calculated bandgap value for undoped CdSe QDs is $2.12 \mathrm{eV}$; its value increases from 2.16 to $2.23 \mathrm{eV}$ as $\mathrm{Zn}$ doping concentration increases and is shown in figure 5 . Since the semiconductor particle size decreases, the electronic bands of the nanomaterial spilt into discrete energy levels followed by bandgap broadening. Also the crystal size reduction leads to confinement of electron-hole motion inside a particle and increase of surface/volume ratio as well.

\subsection{Photoluminescence studies}

Figure 6 compares the PL spectra of CdSe QDs before and after doping of $\mathrm{Zn}$ at different concentrations. The emission wavelengths for undoped and Zn-doped CdSe QDs are presented in table 2. Both undoped and Zn-doped CdSe QDs exhibit red-shift of the absorption wavelengths, resulting in a significant Stokes shift. For undoped CdSe QDs, the luminescence is centred at $603 \mathrm{~nm}$ in the visible region caused by the presence of trap states. These trap states are mostly due to the deficiency of $\mathrm{Cd}^{2+}$ or $\mathrm{Se}^{2-}$ ions on the surface of CdSe QDs [26]. The photoluminescence intensity of Zndoped CdSe QDs increases with Zn concentration; the peak 
Table 2. Energy gap, absorption and emission for undoped and Zn-doped CdSe QDs.

\begin{tabular}{lccc}
\hline $\begin{array}{l}\text { Doping } \\
\text { concentration (\%) }\end{array}$ & $\begin{array}{c}\text { Bandgap } \\
(\mathrm{eV})\end{array}$ & $\begin{array}{c}\text { Absorption } \\
\text { wavelength (nm) }\end{array}$ & $\begin{array}{c}\text { Emission } \\
\text { wavelength (nm) }\end{array}$ \\
\hline CdSe & 2.12 & 583 & 603 \\
CdSe:Zn(1\%) & 2.16 & 574 & 607 \\
CdSe:Zn(2\%) & 2.19 & 566 & 609 \\
CdSe:Zn(3\%) & 2.20 & 562 & 611 \\
CdSe:Zn(4\%) & 2.22 & 558 & 615 \\
CdSe:Zn(5\%) & 2.23 & 555 & 618 \\
\hline
\end{tabular}

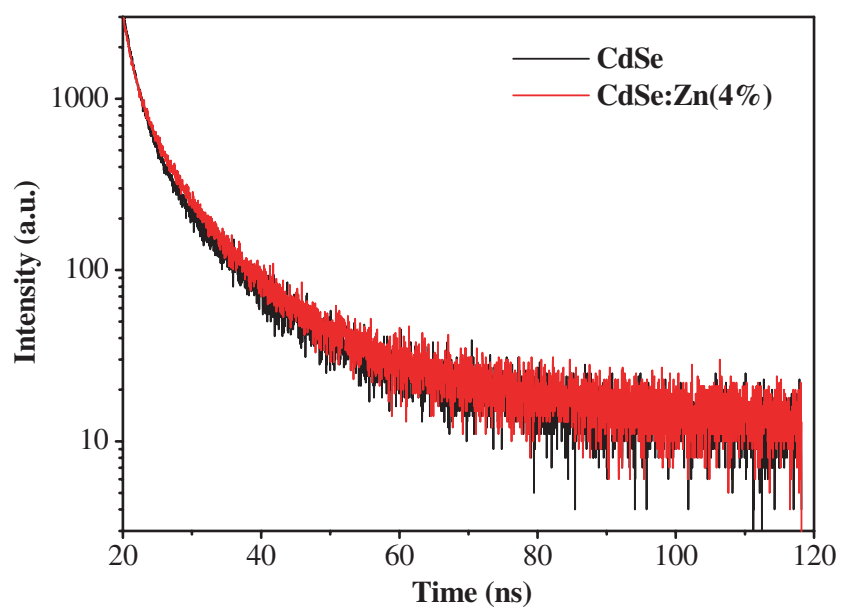

Figure 7. Photoluminescence lifetime spectra of undoped and CdSe:Zn(4\%) QDs.

appears at $615 \mathrm{~nm}$ and red-shifts with $\mathrm{Zn}$ concentration. The intensity reaches the highest when $\mathrm{Zn}$ concentration is $4 \%$. A further increase of $\mathrm{Zn}$ to $5 \%$ results in a decline in the intensity. The recombination of the defects and excitation states induced by $\mathrm{Zn}^{2+}$ provide modified surface trap states, which enhance and shift the luminescence band [27]. The decrease in intensity for $\mathrm{CdSe}: \mathrm{Zn}(5 \%)$ can arise from multiple causes. This is due to the possibility of charge transfer pathways between the CdSe QDs and Zn, thus introducing new nonradiative decay channels, and it would quench the photoluminescence. Also, because of the extremely small diameter, there are many non-radiative trap sites on the surface of CdSe:Zn(5\%) QDs, resulting in lower luminescence.

Further studies like SEM, TEM, FTIR and lifetime analysis were carried out for CdSe:Zn(4\%) QDs due to its high PL intensity and also for undoped CdSe QDs.

\subsection{Photoluminescence lifetime studies}

The lifetime measurements of undoped and CdSe:Zn(4\%) QDs samples at room temperature are shown in figure 7. The measured points were fitted with biexponential curves

$$
I=A_{1} \exp \left(-t / \tau_{1}\right)+A_{2} \exp \left(-t / \tau_{2}\right),
$$

Table 3. Photoluminescence lifetime data of CdSe and $\mathrm{Zn}$-doped CdSe QDs.

\begin{tabular}{lcccccc}
\hline Sample & Size $(\mathrm{nm})$ & $\tau_{1}(\mathrm{~ns})$ & $\tau_{2}(\mathrm{~ns})$ & $A_{1}$ & $A_{2}$ & $\chi^{2}$-value \\
\hline CdSe & 4.4 & 1.6 & 8.7 & 64.18 & 35.82 & 1.3792 \\
CdSe:Zn(4\%) & 3.6 & 1.5 & 8.4 & 56.13 & 43.87 & 1.4656 \\
\hline
\end{tabular}

where $\tau_{1}$ and $\tau_{2}$ are, respectively, the shorter and longer lifetime. $A_{1}$ and $A_{2}$ represent intensity of the decay process with lifetime of $\tau_{1}$ and $\tau_{2}$, respectively, and the values are shown in table 3 . The quality of the fit was very good, which can be evaluated from the $\chi^{2}$-value. The short lifetime $\left(\tau_{1}\right)$ was attributed to the intrinsic electron-hole recombination process of initially populated $\mathrm{CdSe}$ QDs, which is longer than that of bulk CdSe (typically $1 \mathrm{~ns}$ ). This is due to the screening of the radiating field inside CdSe QDs [28]. The observed $\tau_{1}$ values are in good agreement with those of Javier et al [29]. The longer lifetime $\left(\tau_{2}\right)$ was due to the involvement of surface states in the carrier recombination process [30]. The lifetime of $\mathrm{Zn}(4 \%)$-doped CdSe QDs is slightly less than its undoped counterpart. It is already reported that the decay lifetime is inversely proportional to the oscillator strength of a transition and the lifetime is shortened with decreasing size of particles [31]. However, in this case, the shortening of lifetimes in CdSe:Zn(4\%) may be due to the increased density of trap states caused by $\mathrm{Zn}$ dopant in CdSe QDs host and decreased particle size. According to Wang et al, the ratio of $A_{1}$ to $A_{2}$ depends on the quality of QD surfaces. The larger the value of $A_{2}$, the better the surface condition (fewer the surface defects) and the larger the role of surface-related emission [32]. It is observed from table 3 that the value of $A_{2}$ for $\mathrm{CdSe}: \mathrm{Zn}(4 \%)$ is larger than that of undoped CdSe. This indicates that $\mathrm{CdSe}: \mathrm{Zn}(4 \%)$ has fewer surface defects than do undoped CdSe QDs.

\subsection{SEM studies}

SEM images of undoped CdSe and CdSe:Zn(4\%) are shown in figure $8 \mathrm{a}$ and c. From figure 8a, CdSe QDs are observed to be spherical. The CdSe QDs doped with $4 \% \mathrm{Zn}$ are observed to be spherical with a compact and dense structure and increased tendency of agglomeration. The presence of $\mathrm{Cd}$ and Se elements in CdSe QDs and Cd, Se and Zn elements in Zn-doped CdSe QDs was confirmed by EDAX analysis and it is shown in figure $8 b$ and $d$, respectively.

\subsection{TEM studies}

TEM images in figure 9a and c show that the CdSe nanomaterial remains unchanged after doping $\mathrm{Zn}(4 \%)$, suggesting that no significant modification occurred in CdSe nanostructure. Figure $9 \mathrm{~b}$ and $\mathrm{d}$ shows the selected electron diffraction pattern of undoped CdSe and CdSe:Zn(4\%) QDs. The SAED pattern revealed bright continuous concentric rings attributed to the diffraction from the (111), (220) and (311) planes of 

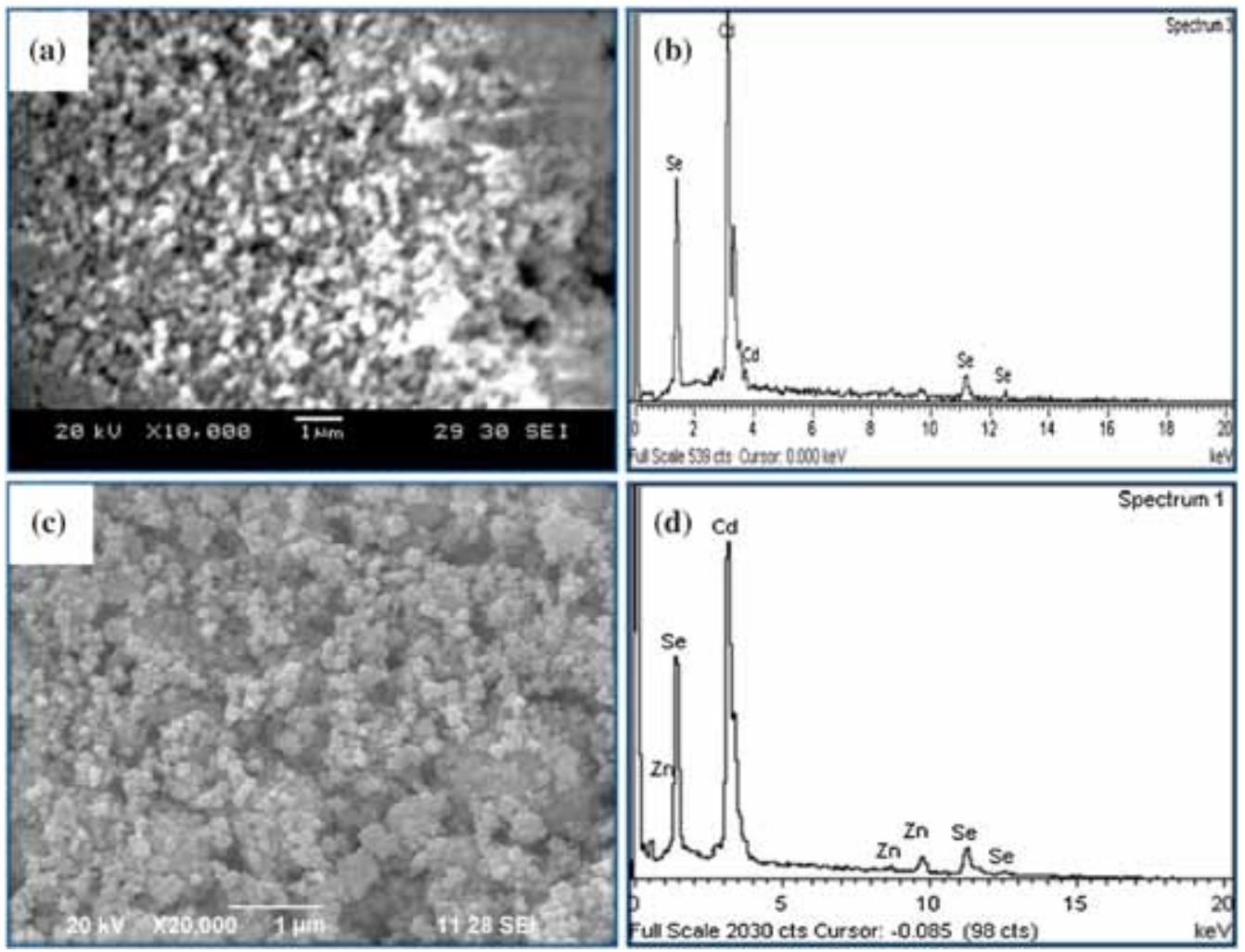

Figure 8. SEM pictures of (a) undoped and (c) Zn(4\%)-doped CdSe QDs and (b, d) corresponding EDAX spectra.
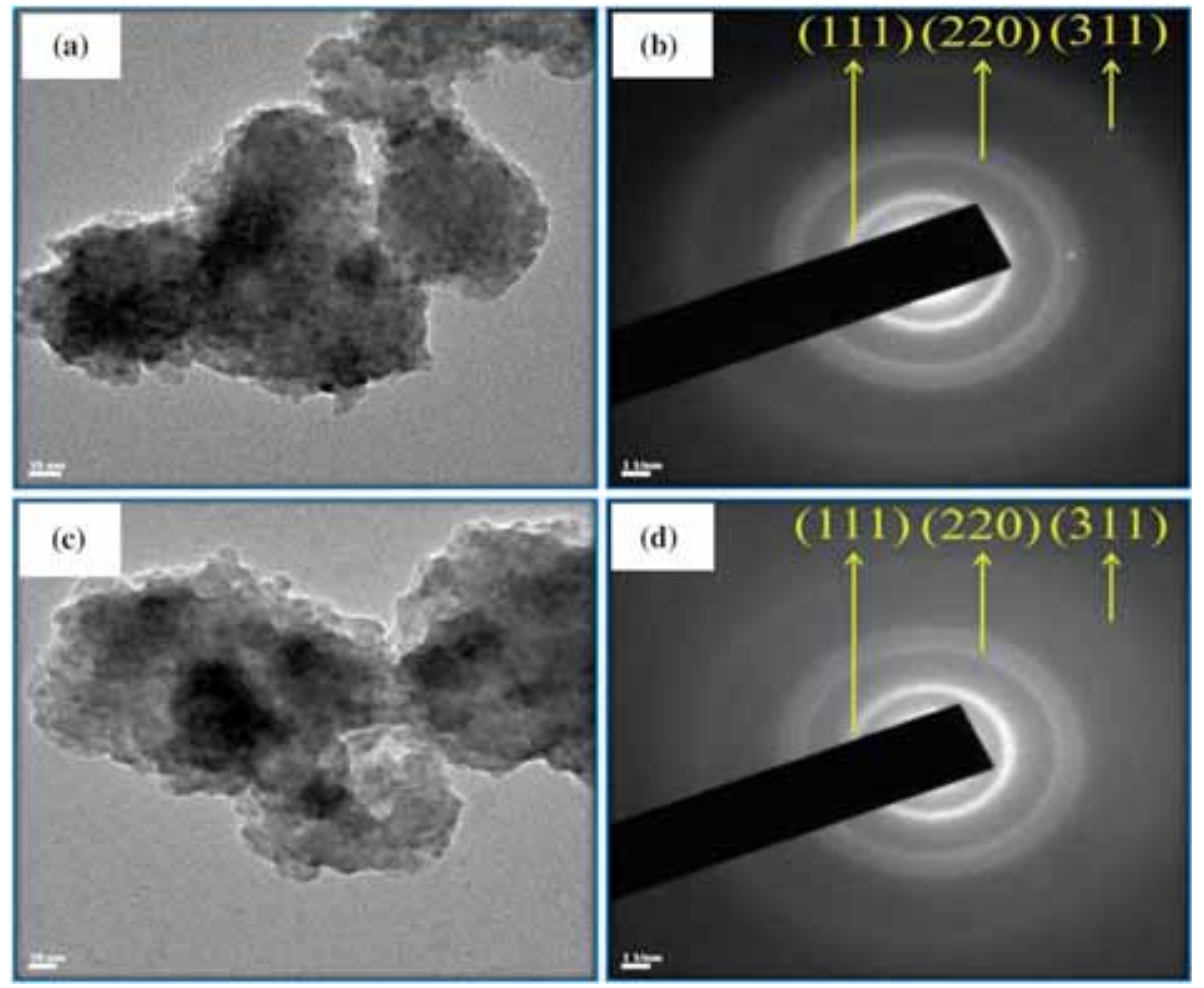

Figure 9. TEM pictures with SAED pattern for (a, b) undoped and (c, d) $\operatorname{Zn}(4 \%)$-doped CdSe QDs. 


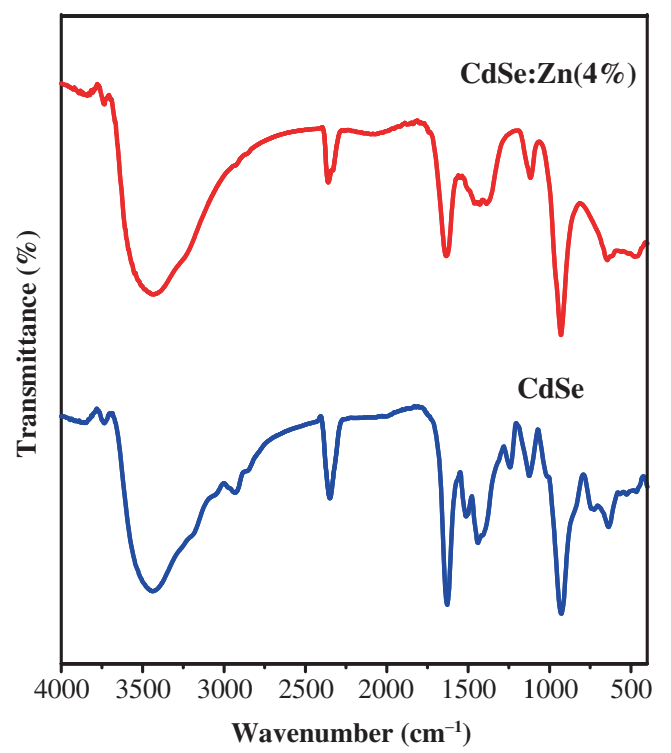

Figure 10. FTIR spectra of undoped and $\mathrm{Zn}(4 \%)$-doped CdSe QDs.

cubic phase in both samples, consistent with XRD data. The particle sizes estimated from TEM images for undoped and $4 \%$-Zn-doped CdSe are 4.4 and $3.7 \mathrm{~nm}$, respectively, close to the size from Scherrer's equation.

\section{$3.7 \quad$ FTIR studies}

Figure 10 shows the FTIR spectra of undoped and $4 \%-\mathrm{Zn}$ doped CdSe QDs. The O-H stretching vibration is observed at $3437 \mathrm{~cm}^{-1}$ for undoped CdSe QDs and $3428 \mathrm{~cm}^{-1}$ for Zn-doped CdSe QDs, which indicates the presence of water molecules on the surface of both samples. The absorption peaks of $\mathrm{O}=\mathrm{C}-\mathrm{H}$ bending vibrations were at 2932 and $928 \mathrm{~cm}^{-1}$ for undoped CdSe QDs and $930 \mathrm{~cm}^{-1}$ for CdSe QDs doped with $4 \% \mathrm{Zn}$. The $\mathrm{CO}_{2}$ molecules were absorbed on both samples which was revealed through the presence of $\mathrm{O}=\mathrm{C}=\mathrm{O}$ stretching vibration at $2349 \mathrm{~cm}^{-1}$ for undoped CdSe QDs and $2358 \mathrm{~cm}^{-1}$ for CdSe QDs doped with $4 \% \mathrm{Zn}$. The $\mathrm{C}=\mathrm{C}$ stretching vibrations were observed at 1628 and $1514 \mathrm{~cm}^{-1}$ for undoped samples and $1634 \mathrm{~cm}^{-1}$ for doped samples. The observed stretching vibrations of $\mathrm{C}=\mathrm{C}$ and $\mathrm{C}-\mathrm{O}$ indicate the presence of acetate ions adsorbed on the surface of both undoped and doped samples. The IR absorption peaks observed at $638 \mathrm{~cm}^{-1}$ for undoped CdSe QDs and $644 \mathrm{~cm}^{-1}$ for CdSe QDs doped with $4 \% \mathrm{Zn}$ may represent the stretching vibrations of $\mathrm{Cd}-\mathrm{Se}$ bond.

\section{Conclusion}

In conclusion, the sizes of synthesized undoped and $\mathrm{Zn}$ doped CdSe QDs are in the range of 4.4-3.5 nm. Both undoped and Zn CdSe QDs were in cubic phase, which was confirmed by XRD. From PL studies, enhanced luminescence is observed at $615 \mathrm{~nm}$ for CdSe QDs doped with $4 \% \mathrm{Zn}$. It is also found that the luminescence decay time for $\mathrm{Zn}(4 \%)$-doped $\mathrm{CdSe}$ QDs is slightly lower than that of undoped CdSe QDs. The obtained QDs are spherical in shape, which was confirmed by TEM analysis. Thus, this mild and operationally simple route may prove to be valuable for nanocrystals-related research.

\section{References}

[1] Sun S, Murrary C B, Weller D, Folks L and Moser A 2000 Science 2871989

[2] Michler P, Imamoglu A, Mason M D, Carson P J, Strouse G F and Buratto S K 2000 Nature 406968

[3] Noh M, Kim T, Lee H, Kim C K, Joo S W and Lee K 2010 Colloids Surf. A: Physicochem. Eng. Aspects 35939

[4] Greenham N C, Peng X G and Alivisatos A P 1996 Phys. Rev. B 5417628

[5] Huynh W U, Peng X G and Alivisatos A P 1999 Adv. Mater. 11923

[6] Screuder M A, Gosnell J D, Smith N J, Warnement M R, Weiss S M and Rosenthal S J 2008 J. Mater. Chem. 18 970

[7] Haldar K K, Sinha G, Lahtinen J and Patra A 2012 ACS Appl. Mater. Interfaces 46266

[8] Selvan S T, Tan T T Y, Yi D K and Jana N R 2010 Langmuir 2611631

[9] Beloglazova N V, Goryacheva I Y, Niessner R and Knop D 2011 Microchim. Acta 175361

[10] Trapiella-Alfonso L, Costa-Fernández J M, Pereiro R and Sanz-Medel A 2011 Biosens. Bioelectron. 264753

[11] Somers R C, Bawendi M G and Nocera D G 2007 Chem. Soc. Rev. 36579

[12] Wang X and Guo X Q 2009 Analyst 134248

[13] Song L and Zhang S 2011 Chem. Eng. J. 166779

[14] Peng Z A and Peng X G 2001 J. Am. Chem. Soc. 123 183

[15] Murray C B, Noms D J and Bawendi M G 1993 J. Am. Chem. Soc. 1158706

[16] Gaponik N, Talapin D V, Rogach A L, Hoppe K, Shevchenko E V, Kornowski A, Eychmüller A and Weller H 2002 J. Phys. Chem. B 1067177

[17] Rong X L, Zhao Q and Tao G H 2002 Chin. Chem. Lett. 23 961

[18] Rogach A L, Negesha D and Ostrander J W 2000 Chem. Mater. 122676

[19] Wang C L, Zhang H and Zhang J H 2007 J. Phys. Chem. C 1112465

[20] Wu Y L, He F, He X W, Li W Y and Zhang Y K 2008 Spectrochim. Acta A $\mathbf{7 1} 1199$

[21] Deng D W, Yu J S and Pan Y J 2006 J. Colloid Interface Sci. 299225

[22] Chang W G, Shen Y H, Xie A J, Zhang H, Wang J and Lu W S 2009 J. Colloid Interface Sci. 335257

[23] Novoselova A V and Lazarev V B 1979 Physicochemical properties of semiconducting materials (Moscow: Nauka) 
[24] Ekimov A I, Hache F, Schanneklein M C, Ricard D, Flytzanis C, Kudryavtsev I A, Yazeva T V, Rodina A V and Efros A L 1993 J. Opt. Soc. Am. B 10100

[25] Chowdhury S, Ahmed G A, Mohanta D, Dolui S K, Avasthi D K and Choudhury A 2005 Nucl. Instr. Meth. Phys. Res. B 240690

[26] Chestnoy N, Harris T D, Hull R and Brus L E 1986 J. Phys. Chem. 903393

[27] Yang P, Lu M K, Song C F, Xu D, Yuan D R, Cheng X F and Zhou G J 2002 Opt. Mater. 20141
[28] Wehrenberg L B, Congjun W and Guyot-Sionnest P $2002 J$. Phys. Chem. B 10610634

[29] Javier A, Magana D, Jennings T and Strouse G F 2003 Appl. Phys. Lett. 831423

[30] Qu L and Peng X 2002 J. Am. Chem. Soc. 1242049

[31] Chen W, Bovin J O, Joly A G, Wang S, Su F and Li G 2004 J. Phys. Chem. B 10811927

[32] Wang X, Qu L, Zhang J, Peng X and Xiao M 2003 Nano Lett. 31103 